\title{
La mujer en cargos de dirección empresarial en los diferentes contextos laborales
}

DOI: https://doi.org/10.33262/ap.v3i2.1.51

\begin{abstract}
(C)
The woman in positions of enterprise direction in the different labor contexts
\end{abstract}

Verónica Elizabeth Villarroel Jácome. ${ }^{1}$ \& Washington Edy Santillán Marroquín. ${ }^{2}$

\begin{abstract}
.
Introduction. The 21 st century will undoubtedly work better, if women had a great participation in the world of work and in the structure of society, which at the moment is in an inequitable state, is poorly designed and supports the consequences of a rationalism and stubborn and inexplicable discrimination. We must firmly emphasize that women are the nucleus of the family, and that the family is the base of society with great potential to generate results in companies. Objective. Determining results are the factors that prevent women from accessing organizational management positions, it is intended in this research, to expose the competitive advantages that it would mean for companies to manage their talents based on policies of equity, justice, diversity and equality of opportunities to achieve business objectives established within a strategic plan. Methodology. This work uses different qualitative and quantitative research techniques for the analysis of the employment situation of women to access positions of power. First, a bibliographic review of the literature will be carried out, which includes various variables that enable or intervene for women to access organizational management positions. Results. Among the most frequent results, (14\%) of female executives occupy management positions in companies (77) cents per dollar are earned by women in relation to men when it comes to salaries and the gap is greater when they

1 Coordinadora de Bienestar Estudiantil del Instituto Superior Tecnológico Bernardo O’Higgins bienestar@instituto-ohiggins.com, https://orcid.org/0000-0002-6291- 505X, Quito- Ecuador.

2 Coordinador Académico del Instituto Superior Tecnológico Bernardo O'Higgins coordinacion.academica@instituto-ohiggins.com, https://orcid.org/0000-0003-1980- 6832, QuitoEcuador.
\end{abstract}


have children . (2.5) times more hours per day women dedicate themselves to unpaid housework and care than men. Conclution. Including women within all the hierarchical structures of the company generates multiple benefits, on the one hand, it would increase the intrinsic motivation in women who have invested in their academic training to occupy these positions and, on the other, the company would acquire an advantage over its competitors, within a market that needs broad business prospects.

Key Words: equity, women, leadership, competitiveness, participation, motivation, communication, market.

\section{Resumen.}

Introducción. El siglo XXI sin duda funcionará mejor, si la mujer tuviera una gran participación en el mundo laboral y en la estructura de la sociedad, la cual por el momento se encuentra en un estado inequitativo, está mal diseñado y soporta las consecuencias de un racionalismo y discriminación tenaz e inexplicable. Debemos acentuar con firmeza que la mujer es el núcleo de la familia, y ésta, la base de la sociedad con un gran potencial para generar resultados en las empresas. Objetivo. Determinar cuáles son los factores que impiden acceder a la mujer a puestos de dirección organizacional, se pretende en esta investigación, exponer las ventajas competitivas que significaría para las empresas gestionar sus talentos basados en políticas de equidad, justicia, diversidad y la igualdad de oportunidades para lograr los objetivos empresariales establecidos dentro de un plan estratégico. Metodología. Este trabajo utiliza diferentes técnicas cualitativas y cuantitativas de investigación para el análisis de la situación laboral de la mujer para acceder a puestos de poder. Se realizará primeramente una revisión bibliográfica de la literatura, en donde incluyen diversas variables que posibilitan o intervienen para que las mujeres accedan a puestos de dirección organizacional. Resultados. Entre los resultados con mayor frecuencia, el (14\%) de las mujeres ejecutivas ocupan cargos de dirección en las empresas (77) céntimos por dólar ganan la mujeres en relación a los hombres cuando se refiere a salarios y la brecha es mayor cuando tienen hijos. $(2,5)$ veces más horas por día las mujeres se dedican a las tareas del hogar y cuidados no remunerados que los hombres. Conclusión. Incluir a las mujeres dentro de todas las estructuras jerárquicas de la empresa genera múltiples beneficios, por un lado, aumentaría la motivación intrínseca en mujeres que han invertido en su formación académica para ocupar estos puestos y por otro, la empresa adquiriría una ventaja sobre sus competidores, dentro de un mercado que necesita amplias perspectivas de negocio.

Palabras Claves: equidad, mujeres, dirección, competitividad, participación, motivación, comunicación, mercado.

\section{Introducción.}

Durante los últimos años ha surgido un enorme interés y preocupación por la limitada presencia de las mujeres en los puestos directivos organizacionales. En la actualidad la 
literatura económica, prensa, revistas de liderazgo e informes sobre responsabilidad social empresarial (RSE) han acuñado el término "Techo de Cristal" para referirse al problema de desigualdad en las esferas de mando de las empresas a favor del género masculino (González, 2015).

Schuh, Hernández, Van Quaquebeke, Hossiep, y Van Dick en 2013 menciona como Joy en 2008 indica el gran avance que ha tenido la mujer, ya que ha alcanzado la paridad de género tanto en su nivel de instrucción como en su participación dentro de la fuerza laboral, sin embargo, en la actualidad aún sigue estando infrarrepresentada en la alta dirección empresarial. Lo que significa que las organizaciones y la sociedad en general soportan un gran desafío ético que deben superar.

Para resolverlo, muchas acciones se han realizado, como por ejemplo en España, la denominada Ley Orgánica 3/2007 que destaca el principio de presencia o composición equilibrada, cuyo objetivo es asegurar que en los cargos de alta responsabilidad en las organizaciones exista una representación paritaria entre hombres y mujeres, es decir que, en su conjunto, las personas de cada sexo no superen el $60 \%$, ni tampoco que la cifra baje del $40 \%$. Una iniciativa muy buena que fomenta claramente el principio de equidad, no obstante, tiene un gran desatino ya que dicha legislación se ha limitado simplemente a recomendar la medida, mas no obliga a que se la cumpla, de este modo no se evidencia ningún cambio importante para resolver el problema (Biedma, 2017).

Por su parte, las empresas conscientes de los cambios vividos en un entorno competitivo y cambiante saben que el desarrollo de líderes del futuro y el aumento de la diversidad promete generar mejores resultados para sus stakeholders, pese a esto, existe aún una falta de énfasis en el desarrollo del liderazgo para las mujeres (O’Neill y Boyle, 2011).

Con el fin de eliminar figuras de desigualdad en los espacios de dirección organizacional muchas empresas han tomado medidas para fortalecer una nueva gestión empresarial, en donde prime un nuevo modelo de creación de valor social conjuntamente con el económico. Permitiendo de esta manera acceder a la mujer a puestos de dirección acorde a su formación académica de forma paritaria con sus homónimos masculinos (González, 2015).

\section{Objetivo General.}

Determinar cuáles son los factores que impiden acceder a la mujer a puestos de dirección organizacional, se pretende en esta investigación, exponer las ventajas competitivas que significaría para las empresas gestionar sus talentos basados en políticas de equidad, justicia, diversidad y la igualdad de oportunidades para lograr los objetivos empresariales establecidos dentro de un plan estratégico.

Con este objetivo general, el trabajo se estructura en tres partes:

Primeramente, la revisión de la literatura donde se expone en primer lugar el concepto "Techo de Cristal", explicando las dificultades que tienen que pasar las mujeres para 
llegar a los tan anhelados puestos de dirección.

Posteriormente mencionaremos sobre los niveles educativos alcanzados y la participación en la vida laboral a la cual ha llegado, sin embargo, pese a todo su potencial y cualificación, en la actualidad todavía se ve perjudicada con la existencia de una brecha de desigualdad de género y sobre todo con los problemas al momento de conciliar su vida familiar y laboral.

También se analizará el tema del liderazgo y las diferencias existentes en relación al género; además, de la importancia del concepto de RSE para la inclusión de la mujer en cargos de dirección.

Finalmente, se indicará las conclusiones más relevantes que se ha obtenido en todo este proceso de investigación y una propuesta de líneas de investigación para el futuro.

\section{Metodologia.}

Este trabajo utiliza diferentes técnicas cualitativas y cuantitativas de investigación para el análisis de la situación laboral de la mujer para acceder a puestos de poder. Se realizará primeramente una revisión bibliográfica de la literatura, en donde incluyen diversas variables que posibilitan o intervienen para que las mujeres accedan a puestos de dirección organizacional.

\section{Resultados.}

\section{El techo de cristal}

La realidad laboral empresarial, refleja que la presencia de las mujeres cada vez cobra mayor notoriedad e importancia para la generación de valor en la industria. Sin embargo, las investigaciones han demostrado que su presencia en los puestos de mayor responsabilidad es insuficiente, debido a la escasez de igualdad de oportunidades en los procesos de desarrollo de carrera que tienen las empresas en comparación con sus homónimos masculinos. Comúnmente, las mujeres se encuentran estancadas en categorías media e inferiores, a pesar de que muchas de ellas cuentan con una formación y capacidades lo suficientemente adecuadas para desempeñar con éxito puestos de alta dirección (Biedma, 2017; Gutiérrez Valdebenito, 2015).

Durante mucho tiempo las mujeres tuvieron que limitarse a cumplir dichas funciones poco valoradas, callando sus ideales, lo que las obligaba a mantener su dependencia económica y emocional hacia sus conyugues.

En este contexto, hace más de 40 años, se empezó a comparar la situación laboral de las mujeres con la metáfora del "El techo de cristal" o "The glass ceiling", acuñada inicialmente en los Estados Unidos, para referirse a las barreras invisibles que permiten a las mujeres visualizar puestos en la cima de su carrera profesional en las organizaciones donde laboran, pero a la vez, obstruyen su paso para alcanzarlas (Simón, 2009). 
Dicha metáfora se sustenta en estereotipos sexistas sociales ambiguos que no son aprobados hoy en día legítimamente, más bien, reflejan un aspecto socio-laboral que ha trascendido durante la historia y que se niega a desaparecer; violentando de esta manera los derechos de desarrollo profesional y de carrera de las mujeres, que experimentan con este fenómeno una gran barrera laboral (Jimeno y Redondo, 2008).

En la actualidad, el ámbito laboral se rodea de una fuerte competencia, en el que las mujeres casi siempre quedan en desigualdad de condiciones con respecto a sus homólogos masculinos, por la intensa carga de los estereotipos que se le adjudican, subestimando su desempeño profesional debido a las responsabilidades socialmente otorgadas, primando siempre el ámbito doméstico sobre el profesional (Moncayo \& Zuluaga, 2015).

Según la OIT, en su informe mundial del 2015 denominado "La mujer en la gestión empresarial: cobrando impulso", indica que en la actualidad existe escases de representación femenina en las cúspides directivas empresariales, lo cual muestra que las barreras invisibles que simbolizan el techo de cristal siguen intactas, pese a la publicidad y a las numerosas movilizaciones en su contra. La realidad laboral refleja que generalmente existe una correlación inversa, ya que cuanto más grande es una empresa menor es el número de mujeres en los puestos más altos; de esta forma, se han acuñado nuevos términos que también identifican la problemática como: los "techos de mármol", "pisos adhesivos", las "paredes de cristal", los "abismos de cristal" y el "progreso glacial", los cuales se comparan con todos los obstáculos que deben enfrentar las mujeres que quieran llegar a puestos de mayor jerarquía empresarial.

En esta misma línea, Ryan y Haslam en 2009 se refiere a este fenómeno como el "acantilado de cristal" para explicar la precariedad laboral que debe enfrentar la mujer cuando ocupa algún puesto de liderazgo. Mencionan además que, las características que lo conforman son muy frecuentes en la sociedad laboral, convirtiéndose en un gran problema a nivel mundial ya que muchas veces son asociados con la crítica y el fracaso en sus puestos de trabajo.

Durante mucho tiempo, la mujer ha tenido que enfrentar varios obstáculos para pretender llegar a una posición de liderazgo y dirección organizacional, la OIT en 2015 resalta dos en particular: primero se refiere al cuestionamiento intrínseco personal sobre sus funciones, así como la percepción que tienen sobre su rol en su círculo familiar, sobre sus obligaciones como madre y esposa; y en segundo lugar, la configuración de la estructura organizacional, en la cual desempeña sus labores profesionales, ya que muchas empresas gestionan su personal femenino y masculino en la ejecución de funciones sociales y económicas de forma diferente.

\section{Niveles educativos femeninos}

Moncayo \& Zuluaga en 2015 mencionan como Giddens en 1987 recalca que, durante muchos años en la historia de nuestra sociedad, el aprendizaje estaba basado en la trasmisión de roles de género, en el cual la familia define normas sociales desde la 
crianza de sus hijos forjando el cumplimiento de estereotipos de género claramente marcados, en los que alienta a la mujer a la ejecución de roles maternales y maritales.

No obstante, la mujer ha demostrado superar la desacreditación social, y para ello, el componente definitivo para su despegue ha sido la formación educativa, de la cual se hizo partícipe y hoy en día demuestra una presencia superior e importante en todos los niveles formativos y académicos (Jimeno y Redondo, 2008).

En el informe de la OIT en 2015 refiere a LA UNESCO la cual anunció que:

“En el mundo el número de mujeres supera al número de hombres que obtienen el primer diploma universitario y que es más probable que las mujeres continúen los estudios universitarios de maestría; representan el 57\% de los diplomados” (pág. 91).

Además, la OIT en 2015 protesta sobre la forma de segregación por sexo de la cual son víctimas las mujeres en los mercados laborales, basados única y exclusivamente en presunciones sociales tradicionales, que coartan las verdaderas habilidades $\mathrm{y}$ capacidades de esta gran fuerza productiva. Pero a pesar de todo ello, los papeles de las mujeres han surgido y se han desarrollado hasta el punto de que hoy por hoy, cada vez más mujeres acceden a opciones educativas a diferentes niveles, incluso superando en número a los hombres.

Por ende, hoy en día ya no pueden tildar a las mujeres de baja cualificación académica para justificar las desigualdades laborales de la cual ha sido siempre protagonista, sobre todo al momento de acceder a puestos de alta dirección, calificándola siempre de poseer menos capacidades, habilidades e inteligencia como se dijo durante muchos siglos (Simón, 2009).

\section{Participación laboral de las mujeres}

En las últimas décadas del siglo XX, surge un gran avance en el desarrollo de los derechos sociales de las mujeres, permitiéndole el acceso a los mercados laborales, aprovechando sus capacidades y habilidades dentro de las sociedades industrializadas.

$\mathrm{Su}$ progresión laboral ha sido firme y creciente, en los que la mujer obtiene muchos avances respecto a igualdad, pese a encontrarse en una sociedad marcadamente machista, lo cual significó una gran fuerza de resistencia. Su lucha no fue fácil, pues incluso las estructuras organizacionales veían en las féminas posibilidades de una carrera profesional corta e interrumpida, debido a que, si en algún momento de su vida llegaran a ser madres, abandonarán su trabajo, por lo tanto, las empresas se muestran negativas a invertir recursos en su formación, lo cual reservan una menor inversión en gran parte de su capital humano (Jimeno y Redondo, 2008).

Desde la inserción a las actividades laborales, las mujeres generalmente han destacado su presencia en profesiones en las que predominan valores de tipo comunal, con la utilización de habilidades interpersonales, en las que prima el servicio y ayuda a los demás, por esta razón desempeñan mayoritariamente actividades dedicadas a la 
enseñanza o la rama sanitaria (López y García, 2009).

La ONU Mujeres en esta línea, menciona la preocupante situación que la mujer tiene que enfrentar por causa de la segregación sectorial y ocupacional (gráfico 1), que enmarca a las mujeres dentro de empleos con menor remuneración y con una mayor precariedad laboral. Además, señala la poca representación que tienen las mujeres en puestos de liderazgo, lo cual impide defender sus derechos laborales fundamentales y asegurar un trabajo digno basado en la equidad.

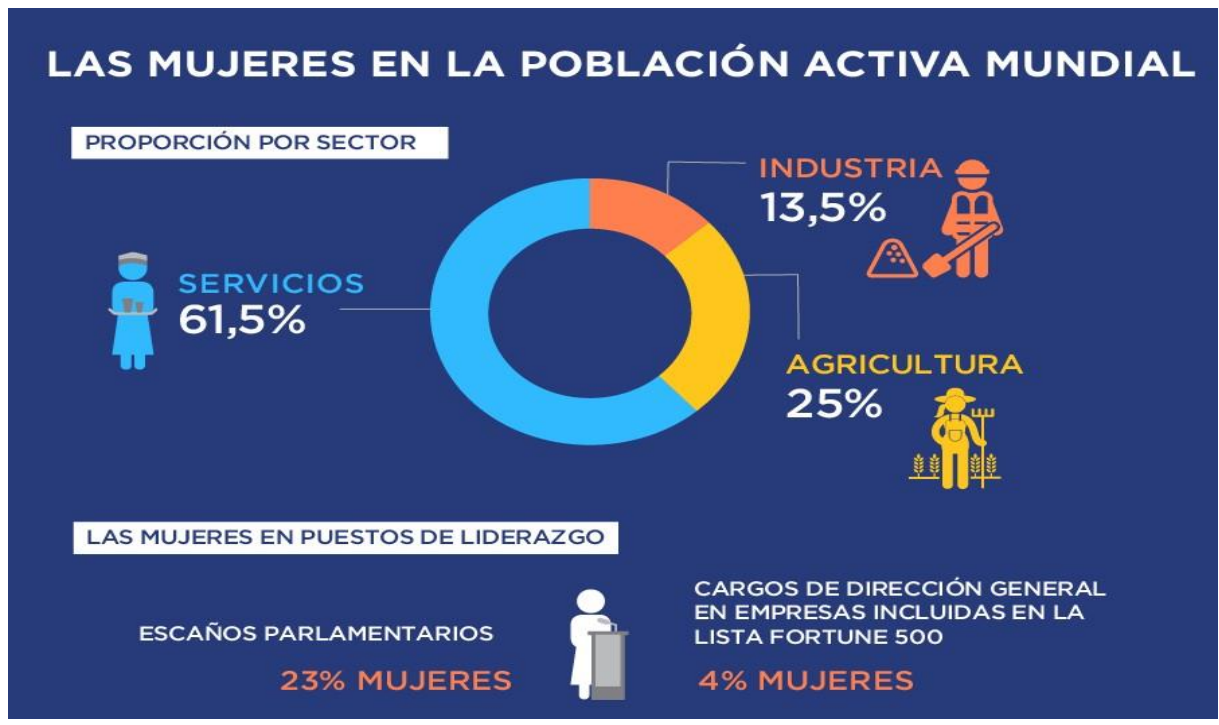

Gráfico 1. Segregación Ocupacional Fuente: (ONU Mujeres)

Así por su parte, López Fernández en 2007 anuncia a Cohen y Huffman que en 2003 se refieren a distintas ocupaciones laborales que han sido enmarcadas por estereotipos sexuales, etiquetándolas como "de hombres" o "de mujeres"; de los cuales siempre generan mayor importancia las actividades realizadas por una figura masculina.

En este sentido, se evidencia una marcada segregación laboral por sexos, en los que se puede diferenciar dos tipos (Rey Medina, 2018):

Segregación laboral horizontal. - también llamado "pared de cristal". Se refiere a las demarcaciones sectoriales realizadas por razones de sexo, a los que le son asignados determinados tipos de ocupaciones para cada uno. Y por lo general, a las mujeres le son atribuidas empleos y sectores con mayor inestabilidad y con menores posibilidades retributivas y de reconocimiento.

Segregación laboral vertical. - en la que exponen las desigualdades de sexo, al momento de distribuir trabajadores en las categorías jerárquicas de una organización. En donde prima la presencia de una figura masculina en los puestos de alta dirección.

López Fernández en 2007 menciona a Wirth que en 2002 señala que paradójicamente, dentro de las denominadas "segregaciones ocupacionales horizontales", donde los empleos son ocupados mayoritariamente por el sexo femenino, se encuentran también inmiscuida la "segregación ocupacional vertical", debido a que es mucho más probable 
que incluso en estos sectores feminizados, lo hombres sean quienes ocupen los puestos de alta dirección y por ende los mejores retribuidos.

No obstante, cabe destacar la importancia de la presencia de la mujer en todos los ámbitos jerárquicos organizacionales, pues los beneficios que esto implica se traduce en aprovechar al máximo todo el talento que la organización posee, reflejando un crecimiento económico a nivel nacional como empresarial, fortaleciendo la idea que la diversidad de género añade valor (OIT, 2015).

\section{Brechas de desigual laboral de género}

Es importante mencionar que la mujer sufre diversas brechas de desigualdad en su vida laboral, no solamente lo concerniente a la brecha salarial, que ha sido la más difundida y protestada, lastimosamente la desigualdad laboral de género abarca mucho más que eso.

En lo referente a la brecha salarial, la ONU Mujeres define este hecho como la diferencia existente en términos de ingresos salariales entre hombres y mujeres. Aduciendo que "a nivel mundial, las mujeres sólo ganan 77 céntimos por cada dólar que ganan los hombres".

Además, enfatiza que esta desigualdad se dispara cuando llegan a ser madres, aumentando consideradamente esta "penalización de la maternidad" (gráfico 2).

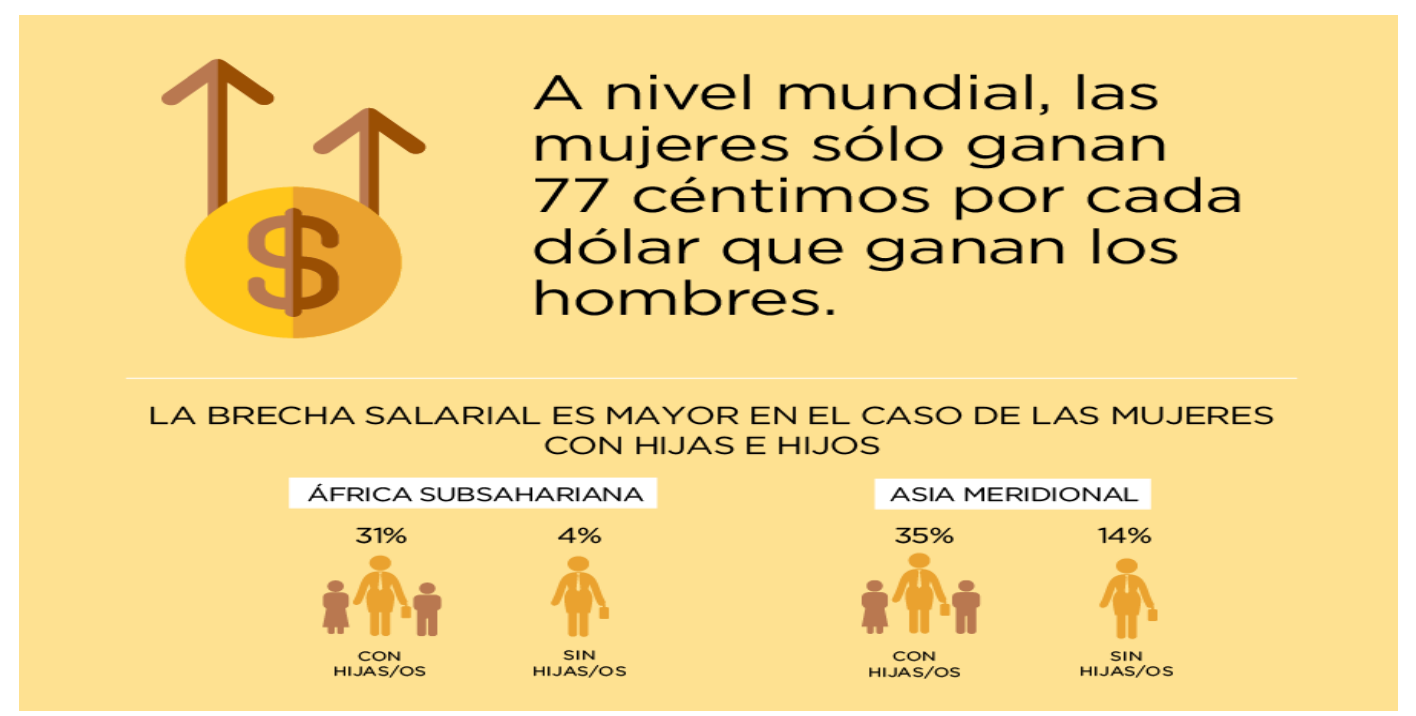

Gráfico 2. Brecha salarial Fuente:(ONU Mujeres)

En este sentido, cabe destacar además la existencia de una marcada brecha en los niveles de participación de liderazgo empresarial, en los que la mujer está escasamente representada. Por ejemplo, Schuh et al. 2013 que citan a Hausman et al. 2010, quienes mencionan que en EEUU el $47 \%$ de la población activa corresponde a las mujeres, pero su fuerza de representación en puestos de dirección solo equivale al $14 \%$ y al $17 \%$ en los escaños del congreso.

Sobre este tema se han realizado muchas investigaciones, cuyas conclusiones fueron 
que, a mayor tamaño de la empresa, menor suele ser la representación femenina en los puestos de dirección, lo cual muestra la desigualdad de oportunidades con las que tienen que vivir las mujeres, pues a pesar de poseer la cualificación requerida no podrán acceder a un desarrollo justo de su carrera profesional (Jimeno y Redondo, 2008).

\section{Conciliación de la vida familiar y laboral}

Desde que la mujer inició su participación en el mercado laboral marcó un cambio social importante, sobre todo en sus roles a desempeñar, debiendo afrontar una doble responsabilidad, ya que nunca dejó de enfrentar las obligaciones familiares que sobre ella pesaban, sobre todo cuando se casaba y tenía hijos (Martínez, Carrasco, Aza, Espinar, Blanco, 2009).

En este sentido La ONU Mujeres, señala cifras en las que demuestra que a nivel mundial las mujeres dedican aproximadamente 2,5 veces más horas por día a las actividades domésticas que los hombres (gráfico 3). Este peso excesivo de trabajo no remunerado, que tienen que llevar las mujeres por el simple hecho de cumplir con su rol de género, no siempre es reconocido ni valorado, pese a su importancia, ya que es uno de los pilares y sustentos de la economía. Más bien, se ha convertido en uno de sus principales obstáculos, impidiendo a las mujeres liberar plenamente su potencial laboral y promover su carrera profesional.

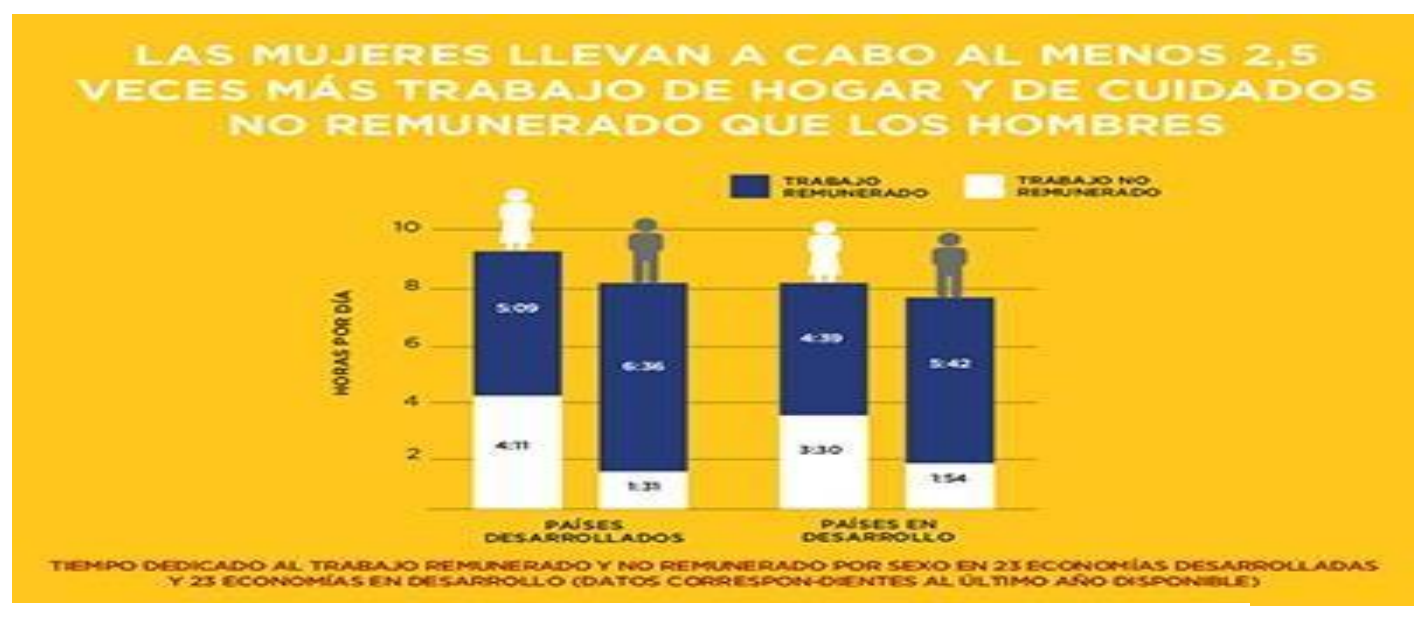

Gráfico 3. Trabajo no remunerado Fuente: (ONU Mujeres)

En esta misma línea, la OIT en 2015 menciona sobre los sacrificios que deben realizar la mayoría de las mujeres que llegan a puestos de dirección, ya que por sus múltiples obligaciones y la cantidad de tiempo que esto les conlleva, reducen drásticamente sus probabilidades de convertirse en madres.

Hoy en día por el tema de la pandemia, las mujeres son las que más sufren realizar teletrabajo ya que cargan doble responsabilidad, no solo la laboral que de por sí es exigente, si no, carga todas las responsabilidades del hogar que implica atención a los hijos y pareja. 


\section{Liderazgo}

Se podría definir a un líder como "un individuo que tiene la capacidad para influir en un colectivo de personas, haciendo que este colectivo trabaje con entusiasmo en el logro de sus objetivos comunes" (García, Ruiz, Gago, López, 2013, p.81).

López y García en 2009 por su parte mencionan que, siempre han estado en contienda dos tipos de liderazgo: primeramente, el transaccional, que hace referencia al uso de recompensas y castigos por parte del líder a sus seguidores, que equivale a una mera relación de intercambio; en contraste a este, se encuentra el liderazgo transformacional, que se refiere a un nuevo estilo marcado por el desarrollo del potencial de sus seguidores, que contribuirá consecuentemente al progreso de la organización, en este contexto, el líder es visto como un modelo de credibilidad, seguridad y en constante proceso de formación e innovación con el fin de establecer metas en pos y mejora de la organización y sobre todo para su equipo de trabajo.

Durante mucho tiempo, las mujeres que pretendía ocupar puestos altos en una jerarquía empresarial, tuvieron que adoptar roles tradicionales de liderazgo de tipo masculino. Posteriormente, el entorno se vuelve más cambiante, y con la aparición de crisis económicas, posibilitan cambiar dicho modelo arcaico hacia un liderazgo transformacional, más cercanos a un rol directivo femenino (Medina Vicent, 2015).

No obstante, O’Neill y Boyle en 2011 mencionan una encuesta realizada por Mercer y un grupo de investigadores, para determinar el nivel de desarrollo de liderazgo femenino en las empresas, determinando que, el $71 \%$ de las organizaciones no tienen una estrategia claramente establecida para desarrollar a las mujeres para ocupar puestos de responsabilidad directiva; esto demuestra que existe una gran falta de interés en el tema por parte de las organizaciones (gráfico 4).

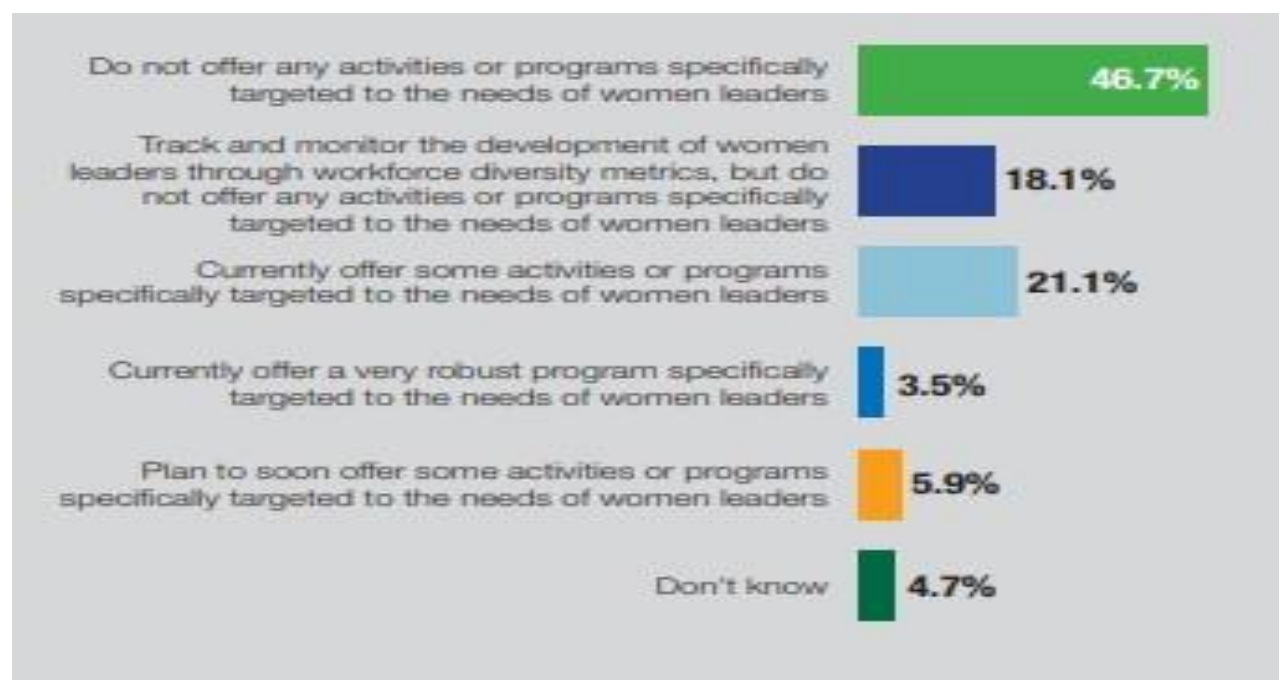

Gráfico 4. Los enfoques de las organizaciones para el desarrollo de mujeres líderes Fuente: tomado de Chief Learning Officer, 2011 


\section{Responsabilidad Social Empresarial}

Según Raufflet, Lozano, Barrera y García en 2012, ponen de manifiesto que el concepto de RSE en el ámbito académico se lo empieza a utilizar muchos años atrás desde 1953, en el libro de Howard Bowen "Social Responsabilities of the Businessman", en donde por primera vez se plantea compendios sobre las responsabilidades que tienen las empresas con respecto a las sociedades donde estas realizan su actividad económica. Pues directa o indirectamente, estas grandes empresas influyen de manera sustancial para que los ciudadanos y las sociedades en sí, se desarrollen o a su vez la destruyan. Por ello la importancia que sus políticas empresariales estén basadas en la conservación y desarrollo social.

En este sentido, uno de los planteamientos que ha respaldado a la RSE, para determinar sus dimensiones, es el modelo de desarrollo sostenible, que entrelaza los aspectos sociales, económicos y medioambientales para lograr una equidad distributiva entre todos sus stakeholders en una organización, donde priorizan actividades como la prestación de servicios sociales, de salud, educación, la participación y la igualdad de género (García, Palacios, Espasandín, 2014).

\section{Responsabilidad Social Corporativa como fuente de ventaja competitiva}

Las dimensiones globales a las que hoy en día ha llegado la praxis de la Responsabilidad Social Empresarial (RSE) en cada una de las organizaciones con y sin fines de lucro aumentan cada vez más. Pero, la forma como cada una de las organizaciones considera que realizan estas acciones, marcan una gran diferencia. Para ello debemos distar las acciones filantrópicas de las verdaderas acciones de RSE, la diferencia radica en que las primeras son obras que la empresa realiza, pero que no están relacionadas con las razón de ser de la misma, están conexas más bien con la caridad, para mejorar la imagen de la empresa ante la sociedad; mientras que las segundas están vinculadas a la gestión estratégica de la empresa y son las acciones que realmente generan una ventaja competitiva sostenible en el mercado (Raufflet, Lozano, Barrera y García, 2012).

\section{Conclusiones.}

- Muchas mujeres tienen la cualificación necesaria como para ocupar puestos de alta dirección en las organizaciones, pese a ello todavía no alcanza la equidad en dichos puestos, debido a que pesa en ellas conceptos estereotipados de género.

- A pesar de ser las mujeres las que representan la mayoría en la obtención de títulos universitarios de estudios superiores en comparación con los hombres, no son consideradas como prioridad para ocupar puestos de dirección organizacional.

- Las evidencias demuestran que existe una marcada brecha de género a favor de los hombres en las tasas de empleo de varios países, sobre todo en Europa donde 
la diferencia es más marcada.

- En lo referente a la segregación laboral horizontal, se puede evidenciar que existen sectores que están feminizados, principalmente en los sectores de la salud, la hotelería y la enseñanza.

- La brecha salarial en todos los países está muy marcada a favor de los hombres.

- Con respecto al uso de tiempo, se ha demostrado que la mujer dedica más tiempo que los hombres en realizar actividades domésticas, cuidado de niños y a la atención de personas dependientes. Aunque estas diferencias son mucho más marcadas en países latinoamericanos donde la carga de trabajo para estas actividades refleja una brecha de género mucho más grande.

- La representación de las mujeres en puestos directivos, refleja que, todavía no existe una cantidad lo suficientemente necesaria como para hablar de igualdad de género.

- Las inversiones en políticas de igualdad de género crean múltiples beneficios tanto en los ingresos económicos de la empresa, como en la calidad de vida laboral de todos sus trabajadores.

- Es importante fomentar por parte de las empresas, que las mujeres aumenten su teletrabajo, de esta manera podrán aumentar las posibilidades de abrirse paso en los ámbitos de dirección empresarial.

- Para aumentar las posibilidades de inserción de la mujer es los altos puestos de responsabilidad organizacional, es fundamental redistribuir las responsabilidades domésticas entre ambos sexos para evitar un exceso de horas de trabajo no remunerado enfocado principalmente hacia las mujeres.

- Las empresas que se basan en políticas de igualdad de género en todos sus niveles jerárquicos organizacionales, pueden convertirse en empresas socialmente responsables, que cuida y desarrolla su capital más importante, sus trabajadores, mejorando su prestigio empresarial ante la sociedad.

\section{Referencias bibliográficas.}

Biedma, J.M. (2017). La mujer directiva. La presencia de la mujer en los consejos de administración de las compañías de IBEX 35. Dossiers Feministes. (22), 13-27. DOI: http://dx.doi.org/10.6035/Dossiers.2017.22.2 Recuperado de http://repositori.uji.es/xmlui/bitstream/handle/10234/168604/Biedma_Ferrer.pdf? seque $\underline{\text { nce }}=1 \&$ is Allowed $=\mathrm{y}$

García, C., Ruiz, E., Gago, C., López, S. (2013). Recursos humanos y responsabilidad social corporativa. Madrid. McGraw-Hill España. Recuperado de https://ebookcentral-proquest-com.bibezproxy.uca.es/lib/bibucascbebooks/reader.action?docID $=3212533$ 
García, J., Palacios, B., Espasandín, F. (2014). Manual práctico de responsabilidad social corporativa. Madrid-España. Ediciones Pirámide.

Gutiérrez Valdebenito, O. (2015). Estudios de liderazgo de hombres y mujeres. Revista Política y Estrategia $\mathrm{N}^{\mathrm{o}} 126$, pp. 133 - 171 DOI: https://doi.org/10.26797/rpye.v0i126.62.

Recuperado de: https://www.politicayestrategia.cl/index.php/rpye/article/view/62/154

INE (s.f.). Mujeres y hombres en España. Recuperado el 22 de mayo del 2019 de: http://www.ine.es/ss/Satellite?L=es_ES\&c=INEPublicacion_C\&cid=125992482 $\underline{2888}$

$\notin p=1254735110672 \&$ pagename $=$ ProductosYServicios\%2FPYSLayout $\&$ param $\underline{1=\mathrm{PY}}$

SDetalleGratuitas.

INE, EUROSTAT. (2017). La vida de las mujeres y los hombres en Europa. Recuperado de https://www.ine.es/prodyser/myhue17/index.html?lang=es

Jimeno, F., Redondo, C., \& Consejo Económico social de la Provincia de Jaén. (2008). La balanza del poder corporativo: El "techo de cristal" en el tejido empresarial de la provincia de Jaén. Jaén: Consejo Económico social de la Provincia de Jaén.

López Fernández, M. (2007). Factores determinantes en el acceso de la mujer a puestos de dirección. Capital Humano, 207: 84-93.

López y García, R. (2009). Situación de las mujeres respecto a posiciones de liderazgo. Jaén. Universidad de Jaén

Martínez, P.,Carrasco, M., Aza, G., Espinar, I, Blanco, A.(2009). Género, empleo y maternidad: análisis comparativo de mujeres con trabajo remunerado y amas de casa. Cuestiones de género: de la igualdad y la diferencia. $\mathrm{N}^{\circ}$ 4, 217-242.

Medina Vicent, M. (2015). Aproximación al estudio del Liderazgo Femenino a través del modelo transformacional. Investigació $i$ gènere a la universitat jaume $i$. Recuperado de http://repositori.uji.es/xmlui/bitstream/handle/10234/158386/Aproximacion_al__ estudi o_del_Liderazgo_Fe.pdf?sequence=1

Moncayo, B. y Zuluaga, D. (2015). Liderazgo y género: barreras de mujeres directivas en la academia. Pensamiento\&Gestión, (39).Recuperadode https://searchproquest-com.bibezproxy.uca.es/docview/1787274723?pq-origsite=summon 
O’Neill, C. \& Boyle, S. (2011). Leadership Challenges for Women at Work. Chief Learning Officer.pp76-78

ONU MUJERES, Empoderamiento económico de la mujer en el cambiante mundo del trabajo.

\section{Recuperado el 20 de mayo del 2019 de: \\ http://www.unwomen.org/es/news/in-focus/csw61}

ONU MUJERES, Las mujeres en el cambiante mundo del trabajo. Algunos datos que deberías conocer. Recuperado el 22 de mayo del 2019 de: http://interactive.unwomen.org/multimedia/infographic/changingworldofwork/e s/index.html

Raufflet, E., Lozano, J., Barrera, E. y García, C. (2012). Responsabilidad Social Empresarial.México. Pearson.

Schuh, C., Hernandez, A., Van Quaquebeke, N., Hossiep, R., Frieg, P., Van Dick, R. (2013). Gender Differences in Leadership Role Occupancy: The Mediating Role of Power Motivation. Springer, pp 363-379, DOI 10.1007/s10551-013-1663-9

SEPE (2018). Informe del Mercado de Trabajo de las Mujeres Estatal Datos 2017. Madrid. Recuperado el 10 de mayo del 2019 de: http://www.sepe.es/contenidos/observatorio/mercado_trabajo/3069-1.pdf

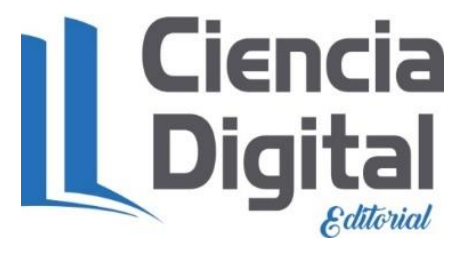




\section{Para citar el artículo indexado}

Villarroel Jácome, V. E., \& Santillán Marroquín, W. E. (2021). La mujer en cargos de dirección empresarial en los diferentes contextos laborales. AlfaPublicaciones, 3(2.1), 85-99. https://doi.org/10.33262/ap.v3i2.1.51

\section{Ciencia LDigital}

El artículo que se publica es de exclusiva responsabilidad de los autores y no necesariamente reflejan el pensamiento de la Revista Alpha Publicaciones.

El artículo queda en propiedad de la revista y, por tanto, su publicación parcial y/o total en otro medio tiene que ser autorizado por el director de la Revista Alpha Publicaciones.
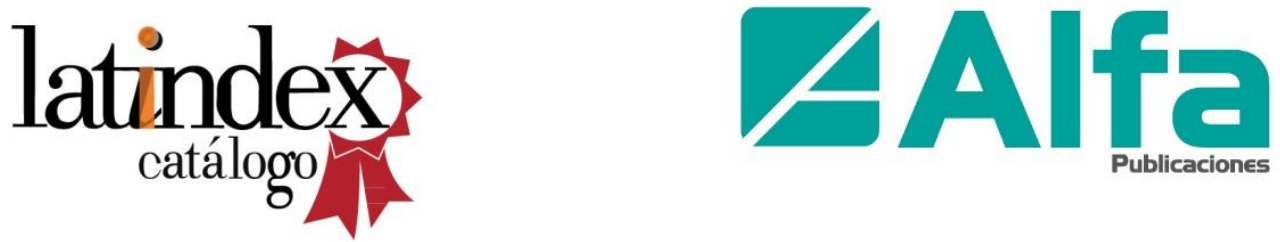